\title{
Professor Werner Hacke scheidet aus dem Herausgebergremium aus
}

\author{
Hugo Van Aken
}

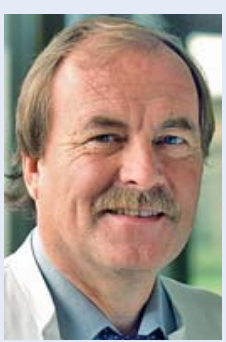

Werner Hacke

Korrespondenzadresse

Prof. Dr. Hugo Van Aken Klinik für Anästhesiologie, operative Intensivmedizin und Schmerztherapie Universitätsklinikum Münster Albert-Schweitzer-

Campus 1, Gebäude A1 48149 Münster hva@uni-muenster.de
Zum Jahresende wird Herr Professor Dr. med. Dr. h.c. Dipl. Psych. Werner Hacke nach 9 Jahren Tätigkeit für die Intensivmedizin up2date aus dem Herausgebergremium ausscheiden. Er hat die Intensivmedizin up2date seit ihrer Gründung im Jahr 2005 als Herausgeber von Seiten der Neurofächer begleitet, geprägt und geformt - dafür gebührt ihm unser herzlicher Dank.

Herr Professor Werner Hacke studierte von 1968 bis 1974 Psychologie und Medizin an der RWTH Aachen. Seine Facharztausbildung absolvierte er im Psychiatrischen Krankenhaus Gangelt, an der Neurologischen Universitätsklinik Aachen und an der Neurologischen Klinik der Universität Bern. Die Promotion erfolgte 1975. Er erlangte seine Facharztanerkennung für Neurologie und Psychiatrie im Jahr 1980. Von 1980 bis 1986 war er Oberarzt an der Neurologischen Klinik der RWTH Aachen, ab 1983, nach seiner Habilitation, bis 1986 leitender Oberarzt und ab 1985 C2-Professor für Neurologie. Von 1986 bis 1987 war er als „Visiting Professor" an der Scripps Clinic and Research Foundation in La Jolla/San Diego (Kalifornien) tätig. Seit 1997 ist er C4-Professor für Neurologie an der Neurologischen Universitätsklinik Heidelberg. Damals war er der jüngste Leiter einer neurologischen Klinik der Geschichte in Deutschland. Er war Dekan der Medizinischen Fakultät Heidelberg von 1989 bis 1991 sowie 1993 bis 1994. Unter anderem hat er etliche klinische Studien zu Prävention (SPACE 1 und 2, CHARISMA, ROCKET-AF) und Therapie (ECASS 1 - 4, DIAS 1,2, DESTINY 1, 2, NEST 3) geleitet und ist korrespondierender Autor der europäischen Leitlinien zur Schlaganfallbehandlung.

Aufgrund seiner hervorragenden wissenschaftlichen Leistungen hat er zahlreiche Ehrenmitgliedschaften und Preise erhalten. Unter anderem ist er Ehrenmitglied der American Neurological Association (ANA) und Ehrenprofessor der Medizinischen Akademie Georgiens. Durch die Staatsuniversität Tbilisi (Georgien) hat er den Titel Dr. h.c. erhalten. Professor Hacke war der
Gründungspräsident der European Stroke Organisation (ESO), zudem war er der erste Vice President der World Federation of Neurology (WFN).

Mit über 330 Originalartikeln und Reviews und einem h-Index von über 100 ist er der weltweit meist zitierte Neurologe und Schlaganfallforscher. Er ist Herausgeber bzw. im Herausgebergremium mehrerer internationaler wissenschaftlicher Zeitschriften wie Neurology, Stroke, Journal of Neurological Sciences, Cerebrovascular Diseases und anderen renommierten Zeitschriften.

Wir hatten das Glück, Herrn Professor Hacke bereits bei der Gründung der Intensivmedizin up2date im Jahre 2005 als Vertreter für die Neurofächer gewinnen zu können. Er hat in all den Jahren ausgesprochen aktiv an der Mitgestaltung der Zeitschrift mitgewirkt und es ist ihm immer gelungen, seine Rubrik mit passenden Themen und hervorragenden Autoren zu besetzen. Als er uns vor einem Jahr mitteilte, dass er mit Beendigung seiner aktiven Laufbahn auch das Herausgebergremium verlassen würde, hat er uns in diesem Zuge Vorschläge für die Zukunft unterbreitet. So haben wir für die Neurofächer Herrn Professor Kollmar (Neurologie) und Herrn Professor Unterberg (Neurochirurgie) in unser Herausgebergremium aufgenommen. Mit beiden hoffen wir nun, die Lücke schließen zu können, die Herr Professor Hacke hinterlässt.

Wir danken ihm ausdrücklich im Namen aller Herausgeber und des Verlags für sein herausragendes Engagement für die Intensivmedizin up2date, für seine Ideen und seine Expertise und wünschen ihm für die Zukunft alles Gute.

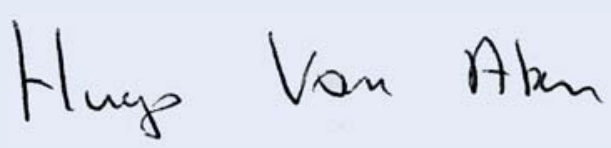

Hugo Van Aken 